\title{
DIGITALCOMMONS
}

—@WAYNESTATE -

Wayne State University

5-1-2007

\section{Existence of Minimizers and Necessary Conditions in Set-Valued Optimization with Equilibrium Constraints}

\author{
Truong Q. Bao \\ Wayne State University \\ Boris S. Mordukhovich \\ Wayne State University, boris@math.wayne.edu
}

\section{Recommended Citation}

Bao, Truong Q. and Mordukhovich, Boris S., "Existence of Minimizers and Necessary Conditions in Set-Valued Optimization with Equilibrium Constraints" (2007). Mathematics Research Reports. Paper 50.

http://digitalcommons.wayne.edu/math_reports/50 


\section{EXISTENCE OF MINIMIZERS AND NECESSARY CONDITIONS IN SET-VALUED OPTIMIZATION WITH EQUILIBRIUM CONSTRAINTS}

TRUONG Q. BAO and BORIS S. MORDUKHOVICH

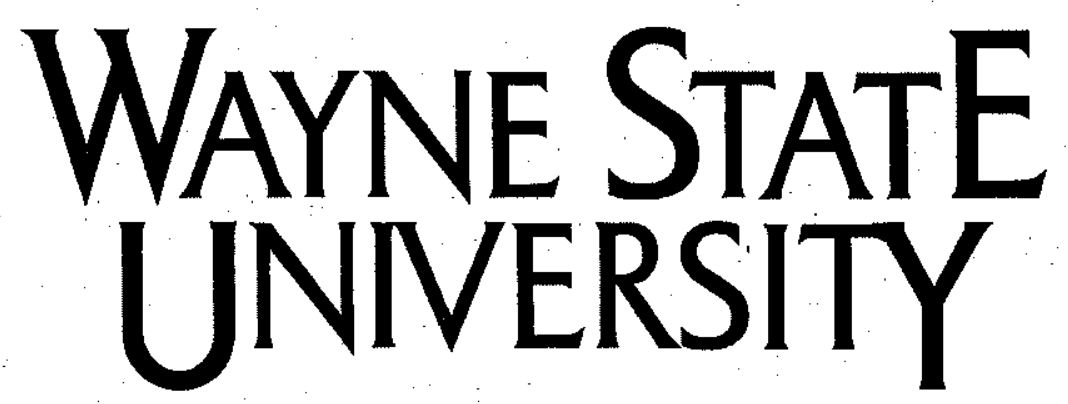

Detroit, MI 48202

Department of Mathematics

Research Report

2007 Series

$\# 5$

This research was partly supported by the National Science Foundation and the Australian Research Council 


\title{
EXISTENCE OF MINIMIZERS AND NECESSARY CONDITIONS IN SET-VALUED OPTIMIZATION WITH EQUILIBRIUM CONSTRAINTS ${ }^{1}$ \\ by
}

\author{
Truong Q. Bao and Boris S. Mordukhovich \\ Department of Mathematics, Wayne State University, Detroit, MI 48202 \\ tqbao@math.wayne.edu, boris@math.wayne.edu \\ Dedicated to Jiři Outrata in honor of his 60th birthday
}

Abstract: In this paper we study set-valued optimization problems with equilibrium constraints (SOPECs) described by parametric generalized equations in the form

$$
0 \in G(x)+Q(x)
$$

where both $G$ and $Q$ are set-valued mappings between infinite-dimensional spaces. Such models particularly arise from certain optimization-related problems governed by set-valued variational inequalities and first-order optimality conditions in nondifferentiable programming. We establish general results on the existence of optimal solutions under appropriate assumptions of the PalaisSmale type and then derive necessary conditions for optimality in the models under consideration by using advanced tools of variational analysis and generalized differentiation.

Keywords: Variational analysis, nonsmooth and set-valued optimization, equilibrium constraints, existence of optimal solutions, necessary optimality conditions, generalized differentiation.

Mathematics Subject Classification 2000: 49J53, 49J52, 90C29, 90A14, 90C33

\section{Introduction}

This paper concerns a general class of set-valued optimization problems whose constraints contain, among others, the so-called equilibrium constraints written in the following form

$$
0 \in G(x)+Q(x)
$$

where both mappings $G: X \rightrightarrows Y$ and $Q: X \Rightarrow Y$ are generally set-valued between Banach (may be finite-dimensional) spaces. Constraints of type (1.1) were first considered by Robinson [16] in the case when the mapping $G=g: X \rightarrow X^{*}$ from $X$ to its dual $X^{*}$ is single-valued and smooth, while $Q: X \rightrightarrows X^{*}$ is set-valued given as the normal cone mapping $Q(x)=N(x ; \Omega)$ to a convex set. Robinson's model of "generalized equations" has been proved to be very convenient and important for both optimization theory and numerous applications. In particular, it covers the classical variational inequalities, complementarity problems, KKT systems in nonlinear programming as well as their extensions and modjfications. It has been subsequently realized that generalized equations provide a natural framework for describing "equilibrium constraints" in various problems of hierarchical optimization and equilibria allowing thus to develop a rich spectrum of theoretical results,

\footnotetext{
${ }^{1}$ This research was partly supported by the National Science Foundation under grants DMS-0304989 and DMS-0603846 and by the Australian Research Council under grant DP-0451168.
} 
numerical methods, and practical applications in the area, which has been unified under the name of Mathematical Programs with Equilibrium Constraints (MPECs).

The monograph by Outrata, Kočvara and Zowe [15] is a pioneering book that, together with that by Luo, Pang and Ralph [6], lays down the foundations of the MPEC theory, algorithms, and applications; see also the more recent books $[4,8]$ and the references therein for further developments. Jiři Outrata is among the first who obtained principal results on optimality conditions and sensitivity analysis for various classes of MPECs employing advanced tools of variational analysis and generalized differentiation (see, e.g., [12, 13]); he is also one of the founders of the new area known now as Equilibrium Problems with Equilibrium Constraints (EPECs); see [14] and also [11] with more discussions and references.

Note that the perturbed version of generalized equations (1.1) particularly needed for their sensitivity analysis is written as

$$
0 \in G(p, u)+Q(p, u)
$$

where $u$ signifies the decision variable and $p$ stands for the perturbation parameter. The majority of previous developments dealt with models of type (1.2) described by single-valued mappings $G=g(p, u)$ depending on parameters and set-valued mappings $Q(u)$ independent of them. Outrata initiated the study of optimization and equilibrium models with equilibrium constraints, where both single-valued and set-valued parts depend on parameters. Such models happen to be important, e.g., in the analysis of quasivariational inequalities; see particularly the recent work [10].

It turns out that many interesting optimization and equilibrium problems important for a variety of applications cannot be adequately described by the generalized equation model (1.1) and its perturbed counterpart (1.2) involving single-valued mappings $G=g$; they require extended versions with both set-valued mappings $G$ and $Q$. The latter classes include, e.g., the so-called set-valued/generalized variational inequalities, variational systems arising in the first-order optimality/KKT conditions for nonsmooth constrained optimization, problems of nondifferentiable bilevel programming, etc. The reader can find more details and references in the recent paper [1] containing necessary optimality conditions for such problems (in both single-objective and vector-objective optimization frameworks) with equilibrium constraints of type (1.2).

The primary goal of this paper is to study set-valued optimization problems with equilibrium constraints of type (1.1) and additional geometric constraints. This class of problems, labeled for brevity as SOPECs, is described by

$$
\begin{array}{ll}
\text { minimize } & F(x) \\
\text { subject to } & 0 \in G(x)+Q(x), \\
& x \in \Omega,
\end{array}
$$

where all the mappings $F: X \rightrightarrows Z, G: X \rightrightarrows Y$, and $Q: X \rightrightarrows Y$ are set-valued between the corresponding Banach spaces, and where $\Omega$ is a nonempty subset of $X$. In this formulation we do not specify the decision-parametric structure of equilibrium constraints as in (1.2) considering the pair $x=(p, u)$ as a single variable under optimization. The main difference 
of problem (1.3) from the one previously studied in [1] is that now we deal with a set-valued cost mapping $F$ whose "minimization" is understood in an appropriate sense; see below.

Furthermore, besides deriving necessary optimality conditions for (1.3), we pay attention in what follows to establishing efficient conditions that ensure the existence of optimal solutions to the set-valued optimization problem formulated above. We have concerned the latter issue in the recent paper [2] for the case of unconstrained problems of set-valued optimization, where the new subdifferential Palais-Smale condition is introduced to provide the existence of weak minimizers. Now we explicitly incorporate the equilibrium and geometric constraint structure of (1.3) into an appropriate extension of the Palais-Smale condition to the SOPECs under consideration. The results obtained in this paper, in both directions of necessary optimality conditions and the existence of optimal solutions to SOPECs in finite-dimensional and infinite-dimensional settings, are strongly based on the generalized differential calculus developed in [7] and particularly applied below to the new subdifferential constructions for set-valued mappings with values in partially ordered spaces.

The rest of the paper is organized as follows. In Section 2 we present and briefly discuss some tools of variational analysis and generalized differentiation needed to derive our main results. We pay a particular attention to subdifferential notions for set-valued mappings with values in partially ordered spaces. Besides the notions introduced in the recent.paper [2], we define here two new modifications different from the previous ones for mappings with values in infinite-dimensional spaces.

Section 3 is devoted to deriving efficient conditions that ensure the existence of optimal solutions to SOPECs while concentrating on the case of weak minimizers. Based on the subdifferential Palais-Smale condition introduced in [2] for unconstrained problems and on appropriate results of generalized differential calculus, we establish verifiable conditions of the Palais-Smale type providing the existence of weak minimizers to multiobjective optimization problems with geometric and equilibrium constraints that are new in both finite-dimensional and infinite-dimensional spaces.

In the final Section 4 we derive pointwise necessary optimality conditions for the SOPECs under consideration with geometric and equilibrium constraints considering both cases of local minimizers and weak local minimizers. Our approach is based on the extremal principle of variational analysis [7] and calculus rules of generalized differentiation. Furthermore, in infinite-dimensional settings we apply the results of $S N C$ calculus [7] ensuring the preservation of the so-called sequential normal compactness (SNC) properties of sets and mappings under appropriate qualification conditions. The latter properties are automatic in finite dimensions while playing a crucial role in infinite-dimensional spaces for both issues of the existence of optimal solutions and necessary optimality conditions studied in the paper. The necessary optimality conditions obtained in Section 4, being new in finite and infinite dimensions, unify and improve various results in single-objective and vector-objective optimization with equality, inequality, operator, and other types of constraints known in the literature; see the discussion in Remark 4.4.

Our notation is basically standard; cf. $[7,17]$. Note that $\mathbb{N}:=\{1,2, \ldots\}, \mathbb{B}$ and $\mathbb{B}^{*}$ stand for the closed unit balls in the space in question and its topological dual, respectively. 
Given a set-valued mapping $F: X \rightrightarrows X^{*}$ between a Banach $X$ and its dual $X^{*}$, the symbol

$$
\begin{aligned}
\operatorname{Limsup}_{x \rightarrow \bar{x}} F(x):=\left\{x^{*} \in X^{*} \mid\right. & \exists \text { sequences } x_{k} \rightarrow \bar{x} \text { and } x_{k}^{*} \stackrel{w^{*}}{\rightarrow} x^{*} \\
& \text { with } \left.x_{k}^{*} \in F\left(x_{k}\right) \text { for all } k \in \mathbb{N}\right\}
\end{aligned}
$$

signifies the sequential Painlevé-Kuratowski upper/outer limit of $F$ at $\bar{x}$ in the norm topology of $X$ and weak* topology $w^{*}$ of $X^{*}$.

\section{Tools of Variational Analysis}

In this section we briefly overview some basic constructions and notions of variational analysis widely used in the paper. We mostly follow the recent book by Mordukhovich [7], where the reader can find more details and references; see also the books by Borwein and Zhu [3] and Rockafellar and Wets [17] for related and additional material. Along with the basic notions, we define in this section new subdifferential constructions for set-valued mappings with values in partially ordered spaces, which - together with those recently introduced in [2]-play a crucial role in formulating and proving the main results of the paper.

Although the definitions presented below hold in arbitrary Banach spaces, the main results of this paper require the Asplund property of the spaces in question; see [7] for the corresponding modifications of the basic constructions in more general settings. Thus, unless otherwise stated, all the primal spaces under consideration are assumed to be Asplund.

Recall that a Banach space $X$ is Asplund if any convex continuous function is densely Fréchet differentiable on $X$. There are many equivalent descriptions of Asplund spaces; see, e.g., $[3,7]$ and the references therein for more discussions and references. Note that the class of Asplund spaces is sufficiently broad including, in particular, every reflective Banach space as well as Banach spaces with separable duals.

We start with generalized normals to nonempty sets. Given $\Omega \subset X$, the Fréchet normal cone (or prenormal cone) to $\Omega$ at $x \in \Omega$ is

$$
\widehat{N}(x ; \Omega):=\left\{x^{*} \in X^{*} \mid \underset{u \stackrel{\Omega}{\rightarrow} x}{\limsup } \frac{\left\langle x^{*}, u-x\right\rangle}{\|x-u\|} \leq 0\right\}
$$

where $u \stackrel{\Omega}{\rightarrow} x$ means that $u \rightarrow x$ with $u \in \Omega$. For convenience put $\widehat{N}(x ; \Omega):=\emptyset$ for $x \notin \Omega$. Then the (basic, limiting, Mordukhovich) normal cone to $\Omega$ at $\bar{x} \in \Omega$ is defined by

$$
N(\bar{x} ; \Omega):=\operatorname{Limsup}_{x \rightarrow \bar{x}} \widehat{N}(x ; \Omega)
$$

via the sequential Painlevé-Kuratowski outer limit (1.4) of Fréchet normals (2.1) as $x \rightarrow \bar{x}$.

A characteristic feature of the basic normal cone (2.2) is its nonconvexity in common situations. In spite of (in fact due to) this nonconvexity, the normal cone (2.2) and the corresponding coderivative and subdifferential constructions generated by it enjoy full calculi in the Asplund space setting; see [7, Chapter 3] for more details.

Let $F: X \rightrightarrows Z$ be a set-valued mapping with the graph

$$
\operatorname{gph} F:=\{(x, z) \in X \times Z \mid z \in F(x)\}
$$


and let $(\bar{x}, \bar{z}) \in \operatorname{gph} F$. Consider two coderivatives of $F$ at $(\bar{x}, \bar{z})$ : the normal coderivative $D_{N}^{*} F(\bar{x}, \tilde{z}): Z^{*} \rightrightarrows X^{*}$ defined by

$$
D_{N}^{*} F(\bar{x}, \bar{z})\left(z^{*}\right):=\left\{x^{*} \in X^{*} \mid\left(x^{*},-z^{*}\right) \in N((\bar{x}, \bar{z}) ; \operatorname{gph} F)\right\}
$$

and the mixed coderivative $D_{M}^{*} F(\bar{x}, \bar{z}): Z^{*} \rightrightarrows X^{*}$ defined by

$$
\begin{aligned}
D_{M}^{*} F(\bar{x}, \bar{z})\left(z^{*}\right):=\left\{x^{*} \in X^{*} \mid\right. & \exists\left(x_{k}, z_{k}\right) \stackrel{\operatorname{gph} F}{\rightarrow}(\bar{x}, \bar{z}), x_{k}^{*} \stackrel{w^{*}}{\rightarrow} x^{*}, z_{k}^{*} \rightarrow z^{*} \\
& \text { with } \left.\left(x_{k}^{*},-z_{k}^{*}\right) \in \widehat{N}\left(\left(x_{k}, z_{k}\right) ; \operatorname{gph} F\right)\right\} .
\end{aligned}
$$

Note that $\bar{z}=f(\bar{x})$ is always omitted in the coderivative notation if $F=f: X \rightarrow Z$ is single-valued. It easily follows from $(2.2)-(2.4)$ that

$$
D_{M}^{*} F(\bar{x}, \bar{z})\left(z^{*}\right) \subset D_{N}^{*} F(\bar{x}, \bar{z})\left(z^{*}\right) \text { for all } z^{*} \in Z^{*}
$$

where the equality holds when, in particular, $\operatorname{dim} Z<\infty$. We have

$$
D_{N}^{*} f(\bar{x})\left(z^{*}\right)=D_{M}^{*} f(\bar{x})\left(z^{*}\right)=\left\{\nabla f(\bar{x})^{*} z^{*}\right\} \text { for all } z^{*} \in Z^{*}
$$

if $f: X \rightarrow Z$ is strictly differentiable at $\bar{x}$; in particular, when $f \in C^{1}$ around this point.

Now we consider a set-valued mapping $F: X \rightrightarrows Z$ between Banach spaces in the case when the range space $Z$ is partially ordered by an nonempty cone $\Theta \subset Z$. In this case we define subdifferential notions for $F$, which-similarly to subdifferentials of real-valued. functions and in contrast to coderivatives of arbitrary mappings as above-depend on the order " $\leq$ " on $Z$ given by the cone $\Theta$ as follows:

$$
z_{1} \leq z_{2} \text { if and only if } z_{2} \in z_{1}+\Theta
$$

Consider the epigraph of $F$ with respect to the ordering cone $\Theta$ defined by

$$
\text { epi } F:=\{(x, z) \in X \times Z \mid z \in F(x)+\Theta\}
$$

with epi $F=\operatorname{gph} F$ if $\Theta=\{0\}$ and the strict inclusion $\operatorname{gph} F \cdot \subset$ epi $F$ holding otherwise; we omit $\Theta$ in the epigraph notation for simplicity. Adopting the approach in [2], introduce the following four subdifferential constructions for $F$ needed to formulate and justify the main results of this paper; only two of them have been defined in [2]. These subdifferentials are generated by the corresponding coderivatives (2.3) and (2.4) of the epigraphical multifunction $\mathcal{E}_{F}: X \rightrightarrows Z$ associated with $F$ (and $\Theta$ ) by

$$
\mathcal{E}_{F}(x):=\{z \in Z \mid z \in F(x)+\Theta\}
$$

Definition 2.1 (normal and mixed subdifferentials of set-valued mappings). Let $F: X \rightrightarrows Z$ with $Z$ partially ordered by a cone $\Theta$, and let $(\bar{x}, \bar{z}) \in$ epi $F$. Then:

-The NORMAL SUBDIFFERENTIAL of $F$ at $(\bar{x}, \bar{z})$ is

$$
\partial_{N} F(\bar{x}, \bar{z}):=\left\{x^{*} \in X^{*} \mid x^{*} \in D_{N}^{*} \mathcal{E}_{F}(\bar{x}, \bar{z})\left(z^{*}\right),-z^{*} \in N(0 ; \Theta), \quad\left\|z^{*}\right\|=1\right\} .
$$


-The MIXED SUBDIFFERENTIAL of $F$ at $(\bar{x}, \bar{z})$ is

$$
\partial_{M} F(\bar{x}, \bar{z}):=\left\{x^{*} \in X^{*} \mid x^{*} \in D_{M}^{*} \mathcal{E}_{F}(\bar{x}, \bar{z})\left(z^{*}\right), \quad-z^{*} \in N(0 ; \Theta),\left\|z^{*}\right\|=1\right\} .
$$

-The NORMAL SINGULAR SUBDIFFERENTIAL and the MIXED SINGULAR SUBDIFFERENTIAL of $F$ at $(\bar{x}, \bar{z})$ are defined, respectively, by

$$
\partial_{N}^{\infty} F(\bar{x}, \bar{z}):=D_{N}^{*} \mathcal{E}_{F}(\bar{x}, \bar{z})(0) \text { and } \partial_{M}^{\infty} F(\bar{x}, \bar{z}):=D_{M}^{*} \mathcal{E}_{F}(\bar{x}, \bar{z})(0) .
$$

Clearly, there is no difference between the normal and mixed subdifferentials introduced, as well as between their singular counterparts, if $\operatorname{dim} Z<\infty$. However, they may be essentially different in infinite dimensions. In the case of extended-real-valued functions $\varphi: X \rightarrow(-\infty, \infty]$, the subdifferentials $(2.5)-(2.7)$ reduce, with $\Theta=R_{+}$, to the corresponding subdifferential constructions by Mordukhovich; see [7].

In what follows we employ the subdifferentials (2.5)-(2.7) of set-valued mappings to the set-valued optimization problems under consideration. Our approach is largely based, due to the above definitions, on the extended normal cone and coderivative calculus rules for the limiting constructions involved. To proceed in this way in infinite-dimensional settings, we need to use appropriate "sequential normal compactness" properties for sets and set-valued mappings with values in partially ordered spaces.

Recall that a set $\Omega \subset X \times Z$ is sequentially normally compact (SNC) at $(\bar{x}, \bar{z}) \in \Omega$ if for any sequences of elements $\left(x_{k}, z_{k}, x_{k}^{*}, z_{k}^{*}\right) \in X \times Z \times X^{*} \times Z^{*}$ satisfying

$$
\left(x_{k}, z_{k}\right) \stackrel{\Omega}{\rightarrow}(\bar{x}, \bar{z}) \text { and }\left(x_{k}^{*}, z_{k}^{*}\right) \in \widehat{N}\left(\left(x_{k}, z_{k}\right) ; \Omega\right), \quad k \in \mathbb{N},
$$

one has the implication $\left(x_{k}^{*}, z_{k}^{*}\right) \stackrel{w^{*}}{\rightarrow}(0,0) \Longrightarrow\left\|\left(x_{k}^{*}, z_{k}^{*}\right)\right\| \rightarrow 0$ as $k \rightarrow \infty$. The more subtle partial $S N C$ property of $\Omega$ at $(\bar{x}, \bar{z})$ means that for any sequences $\left(x_{k}, z_{k}, x_{k}^{*}, z_{k}^{*}\right)$ satisfying (2.8) one has the implication

$$
\left[x_{k}^{*} \stackrel{w^{*}}{\rightarrow} 0,\left\|z_{k}^{*}\right\| \rightarrow 0\right] \Longrightarrow\left\|x_{k}^{*}\right\| \rightarrow 0 \text { as } k \rightarrow \infty .
$$

We refer the reader to the books $[7,8]$ for efficient conditions ensuring the fulfillment of these properties (which are clearly automatic in finite dimensions) and their preservation under various operations. The main results of this paper require the following modifications of the above properties in the case of mappings with values in partially ordered spaces.

Given $F: X \rightrightarrows Z$ with the range space $Z$ ordered by a cone $\Theta$, we say that $F$ is sequentially normally epi-compact (SNEC) at $(\bar{x}, \bar{z}) \in$ epi $F$ if its epigraph generated by $\Theta$ is SNC at this point. Correspondingly, the partially $S N E C$ property of $F$ at $(\bar{x}, \bar{z})$ is induced in the same way by the partial SNC property of the epigraph epi $F$ at $(\bar{x}, \bar{z})$.

It turns out that the above SNC/SNEC properties are ensured by certain Lipschitzian behavior of sets and mappings; cf. [7, Chapter 1]. In particular, $F$ is partially SNEC at $(\bar{x}, \bar{z})$ if it is epi-Lipschitz-like (ELL) around this point, i.e., there are neighborhoods $U$ of $\bar{x}$ and $V$ of $\bar{z}$ and a constant $\ell \geq 0$ such that

$$
\mathcal{E}_{F}(x) \cap V \subset \mathcal{E}_{F}(u)+\ell\|x-u\| \mathbb{B} \text { for all } x, u \in U .
$$


The latter means in fact that the epigraphical multifunction $\mathcal{E}_{F}$ associated with $F$ enjoys the Lipschitz-like/Aubin property around $(\bar{x}, \bar{z})$; cf. $[7,17]$.

Furthermore, it follows from [7, Theorem 1.44] and the constructions of $\partial_{M}^{\infty} F$ in (2.7) that the ELL property of $F$ around $(\bar{x}, \bar{z})$ implies that

$$
\partial_{M}^{\infty} F(\bar{x}, \bar{z})=\{0\}
$$

The major driving force of the underlying generalized differential and SNC calculus results mentioned above is the following Extremal Principle of variational analysis (see particularly [7, Chapter 2] and both volumes [7, 8] for a variety of applications), which plays a' crucial role in deriving the main results of this paper. Recall that $\bar{x} \in \Omega_{1} \cap \Omega_{2}$ is a local extremal point of the set system $\left\{\Omega_{1}, \Omega_{2}\right\}$ in $X$ if there exists a neighborhood $V$ of $\bar{x}$ such that for any $\varepsilon>0$ we can find $a \in \varepsilon \mathbb{B}$ with

$$
\Omega_{1} \cap\left(\Omega_{2}+a\right) \cap V=\emptyset .
$$

The Extremal Principle. Let $\bar{x}$ be a local extremal point of the set system $\left\{\Omega_{1}, \Omega_{2}\right\}$, where both $\Omega_{1}$ and $\Omega_{2}$ are locally closed around $\bar{x}$. Then for every $\varepsilon>0$ there are

$$
x_{i} \in \Omega_{i} \cap(\bar{x}+\varepsilon \mathbb{B}) \text { and } x_{i}^{*} \in \widehat{N}\left(x_{i} ; \Omega_{i}\right)+\varepsilon \mathbb{B}^{*}, \quad i=1,2,
$$

satisfying the relationships

$$
\left\|x_{1}^{*}\right\|+\left\|x_{2}^{*}\right\|=1 ; \quad x_{1}^{*}+x_{2}^{*}=0 .
$$

\section{Existence of Optimal Solutions to SOPECs}

In this section we formulate set-valued optimization problems in the presence of geometric and equilibrium constraints and derive verifiable conditions for the existence of optimal solutions to these problems using the tools of generalized differentiation discussed in Section 2. Without further mentioning, suppose that the constrained problems under consideration have nonempty sets of feasible solutions.

Let $Z$ be Banach space ordered by a cone $\Theta \neq\{0\}$, which is always assumed to be closed, convex, and pointed in the sequel. Given a set $\Lambda \subset Z$ and following the book by Jahn [5], we say that $\bar{z} \in \Lambda$ is a minimal point of $\Lambda$ if

$$
\Lambda \cap(\bar{z}-\Theta)=\{\bar{z}\}
$$

The collection of minimal points of $\Lambda$ is equivalently described by

$$
\operatorname{Min} \Lambda:=\{\bar{z} \in \Lambda \mid \bar{z}-z \notin \Theta \text { whenever } z \in \Lambda\} \text {. }
$$

If int $\Theta \neq \emptyset$, we similarly define weak minimal points $\bar{z}$ of $\Lambda$ by

$$
\Lambda \cap(\bar{z}-\operatorname{int} \Theta)=\emptyset
$$

Given $F: X \rightrightarrows Z$ and $\Xi \subset X$, we start with the following set-valued optimization problem under arbitrary geometric constraints:

$$
\text { minimize } F(x) \text { subject to } x \in \Xi
$$


and say that $(\bar{x}, \bar{z}) \in \operatorname{gph} F$ is a minimizer to (3.1) if $\bar{x} \in \Xi$ and $\bar{z}$ is a minimal point of the image set $F(\Xi):=\cup_{x \in \Xi} F(x)$, i.e.,

$$
F(\Xi) \cap(\bar{z}-\Theta)=\{\bar{z}\}
$$

Similarly, $(\bar{x}, \bar{z}) \in \operatorname{gph} F$ is a weak minimizer to (3.1) with int $\Theta \neq \emptyset$ if $\bar{x} \in \Xi$ and $\bar{z}$ is a weak minimal point of $F(\Xi)$, i.e.,

$$
F(\Xi) \cap(\bar{z}-\operatorname{int} \Theta)=\emptyset
$$

In the first result of this section we establish verifiable conditions ensuring the existence of weak minimizers to problem (3.1) developing the corresponding result of [2, Theorem 4.3] on the existence of weak minimizers to the unconstrained problem of minimizing $F$.

Considering a set-valued mapping $F: X \rightrightarrows Z$ and a set $\Xi \subset X$ as in (3.1), we say that: $F$ is quasibounded from below with respect to $\Xi$ if there is a bounded set $M$ such that

$$
F(\Xi) \subset M+\Theta
$$

Correspondingly, a set $A \subset Z$ is quasibounded from below if the constant mapping $F(x) \equiv A$ has this property. Following [2], we say that $F$ satisfies the subdifferential Palais-Smale condition if any sequence $\left\{x_{k}\right\} \subset X$ such that

$$
\text { there are } z_{k} \in F\left(x_{k}\right) \text { and } x_{k}^{*} \in \partial_{N} F\left(x_{k}, z_{k}\right) \text { with }\left\|x_{k}^{*}\right\| \rightarrow 0 \text { as } k \rightarrow \infty
$$

contains a convergent subsequence provided that $\left\{z_{k}\right\}$ is quasibounded from below.

Our goal now is to introduce an appropriate analog of the subdifferential Palais-Smale condition involving the cost mapping $F$ and the constraint set $\Xi$ in (3.1) that guarantees the existence of weak minimizers to this constrained problem. The following one obtained from (3.4) by using subdifferential calculus meets this purpose.

Definition 3.1 (Palais-Smale condition in set-valued optimization with geometric constraints). We say that the Palais-Smale condition holds in (3.1) if any sequence $\left\{x_{k}\right\} \subset \Xi$ as $k \rightarrow \infty$ such that

$$
\text { there are } z_{k} \in F\left(x_{k}\right) \text { and } x_{k}^{*} \in \partial_{N} F\left(x_{k}, z_{k}\right)+N\left(x_{k} ; \Xi\right) \text { with }\left\|x_{k}^{*}\right\| \rightarrow 0
$$

contains a convergent subsequence provided that $\left\{z_{k}\right\}$ is quasibounded from below.

The next theorem ensures the existence of weak minimizers to the constrained problem (3.1) under the Palais-Smale condition from Definition 3.1 combined with appropriate qualification and $S N C$ assumptions imposed on the initial data.

Theorem 3.2 (existence of weak minimizers in set-valued optimization with geometric constraints). Let $F \rightrightarrows X \rightarrow Z$ be quasibounded from below with respect to $\Xi$ and have the closed epigraph, let the sets $\Xi$ and $\operatorname{Min} F(x)$ as $x \in \Xi$ be closed, and let

$$
\text { for every } x \in \Xi \text { and } z \in F(x) \text { there is } \bar{z} \in \operatorname{Min} F(x) \text { with } \bar{z} \leq z \text {. }
$$


Assume in addition that the Palais-Smale condition from Definition 3.1 holds and that for every $(x, z) \in \operatorname{gph} F$ with $x \in \Xi$ one has the following:

(a) either $F$ is partially SNEC at $(x, z)$, or $\Xi$ is SNC at $x$;

(b) the pair $\{F, \Xi\}$ satisfies the qualification condition

$$
\partial_{M}^{\infty} F(x, z) \cap(-N(x ; \Xi))=\{0\}
$$

Then problem (3.1) admits a weak minimizer.

Proof. Consider the restriction of $F$ to $\Xi$ given by

$$
F_{\Xi}(x):=F(x)+\Delta(x ; \Xi) \text { with } \Delta(x ; \Xi):= \begin{cases}0 \in Z & \text { if } x \in \Xi \\ \emptyset & \text { otherwise. }\end{cases}
$$

Obviously the constrained problem (3.1) is equivalent to the unconstrained problem of minimizing the restriction $F_{\Xi}$ over $X$. Applying [2, Theorem 4.3] to the latter unconstrained problem, we get the existence of weak minimizers to it-and hence to (3.1)-if $F_{\Xi}$ satisfies the afore-mentioned subdifferential Palais-Smale condition for unconstrained minimization. Due to the structures of (3.4) with $F=F_{\Xi}$ and of (3.5), the subdifferential Palais-Smale condition for the unconstrained problem follows from the one in Definition 3.1 provided the fulfillment of the calculus rule

$$
\partial_{N} F_{\Xi}(x, z) \subset \partial_{N} F(x, z)+N(x ; \Xi)
$$

To justify (3.9) for the normal subdifferential (2.5), we use its definition and the basic intersection rule in product spaces derived in [7, Theorem 3.4] from the extremal principle. Applying this rule to the set intersection

$$
\text { epi } F_{\Xi}=\Omega_{1} \cap \Omega_{2} \text { with } \Omega_{1}:=\operatorname{epi} F \text { and } \Omega_{2}:=\Xi \times Z \text {, }
$$

and taking into account the structures of $\Omega_{i}$ in (3.10) as well as the simple relationship

$$
\partial_{N} \Delta(x ; \Xi)=N(x ; \Xi) \text { for any } x \in \Xi,
$$

we arrive at (3.9) under the fulfillment of the SNEC/SNC and qualification conditions imposed in the theorem. This completes its proof.

It turns out that both the qualification and partially SNEC conditions of the theorem are automatic for a broad class of ELL set-valued mappings defined in Section 2.

Corollary 3.3 (existence of weak minimizers for constrained minimization of ELL mappings). Let the cost mapping $F$ be ELL around any point $(x, z) \in \operatorname{gph} F$ with $x \in \Xi$ under the general assumptions of Theorem 3.2. Then there exist weak minimizers to problem (3.1) provided the fulfilment of the Palais-Smale condition from Definition 3.1 .

Proof. This follows from relationship (2.9) for ELL mappings and the fact that such mappings are always SNEC around the points in question; see Section 2.

Next we establish an existence theorem for the principal set-valued optimization problem of this paper with both geometric and equilibrium constraints formulated in (1.3). To derive 
this result, we reduce the general SOPEC (1.3) to a set-valued optimization problem with only the geometric constraint of type (3.1) considered in Theorem 3.2. Such a reduction procedure allows us to obtain verifiable conditions for the existence of weak minimizers to problem (1.3) in terms of the initial data of this problem-mainly due to extended calculus rules available for the generalized differential constructions and SNC properties involved in the major conditions of Theorem 3.2.

Theorem 3.4 (existence of weak minimizers to SOPECs). Let the sets epi $F$, gph $G$, gph $Q$, and $\Omega$ in (1.3) be closed, let

$$
\Xi:=\operatorname{gph} G \cap \operatorname{gph}(-Q) \cap(\Omega \times Y),
$$

and let the mapping $\widetilde{F}(x, y):=F(x)$ satisfy condition (3.6) relative to set (3.11) with the closed minimum set $\operatorname{Min} F(x)$; the latter is automatic when the corresponding condition (3.6) of Theorem 3.2 is satisfied for the cost mapping $F$ in (1.3) with respect to the geometric constraint set $\Omega$. Furthermore, assume that the following hold:

(a) The two QUALIFICATION CONDITIONS

$$
\begin{gathered}
\left(-\partial_{M}^{\infty} F(x, z)\right) \cap\left(D_{N}^{*} G(x, y)\left(y^{*}\right)+D_{N}^{*} Q(x,-y)\left(y^{*}\right)+N(x ; \Omega)\right)=\{0\} \\
{\left[\begin{array}{l}
x_{1}^{*} \in D_{N}^{*} G(x, y)\left(y^{*}\right), x_{2}^{*} \in D_{N}^{*} Q(x,-y)\left(y^{*}\right) \\
x_{3}^{*} \in N(x ; \Omega), x_{1}^{*}+x_{2}^{*}+x_{3}^{*}=0
\end{array}\right] \Longrightarrow\left[\begin{array}{l}
y^{*}=0 \\
x_{1}^{*}=x_{2}^{*}=x_{3}^{*}=0
\end{array}\right]}
\end{gathered}
$$

whenever $x \in \Omega, z \in F(x), y \in G(x) \cap(-Q(x))$, and $y^{*} \in Y^{*}$.

(b) The SOPEC PALAIS-SMALE CONDITION: any sequence $\left\{x_{k}\right\} \subset \Omega$ such that

there are $z_{k} \in F\left(x_{k}\right), y_{k} \in G\left(x_{k}\right) \cap\left(-Q\left(x_{k}\right)\right), y_{k}^{*} \in Y^{*}$, and $\left\|x_{k}^{*}\right\| \rightarrow 0$ with $x_{k}^{*} \in \partial_{N} F\left(x_{k}, z_{k}\right)+D_{N}^{*} G\left(x_{k}, y_{k}\right)\left(y_{k}^{*}\right)+D_{N}^{*} Q\left(x_{k},-y_{k}\right)\left(y_{k}^{*}\right)+N\left(x_{k} ; \Omega\right)$

contains a convergent subsequence provided that $\left\{z_{k}\right\}$ is quasibounded from below.

(c) The SNC CONDITIONS for any $x \in \Omega, z \in F(x)$ and $y \in G(x) \cap(-Q(x))$ :

-either $F$ is partially $S N E C$ at $(x, z)$ and all but one of the sets $\operatorname{gph} G, \operatorname{gph} Q$, and $\Omega$ are $S N C$ at $(x, y),(x,-y)$, and $x$, respectively;

-or all the sets $\operatorname{gph} G, \operatorname{gph} Q$, and $\Omega$ are $S N C$ at $(x, y),(x,-y)$, and $x$, respectively.

Then the SOPEC (1.3) admits a weak minimizer.

Proof. It is easy to observe that the SOPEC problem (1.3) is equivalent to the set-valued optimization problem (3.1) involving only the geometric constraint given by the set $\Xi$ from (3.11) and the cost mapping $\widetilde{F}$ defined in the formulation of the theorem:

$$
\text { minimize } \widetilde{F}(x, y) \text { subject to }(x, y) \in \Xi \subset X \times Y \text {. }
$$

Furthermore, the constraint set $\Xi$ from (3.11) in the latter problem is represented as the intersection $\Xi=\Omega_{1} \cap \Omega_{2} \cap \Omega_{3}$, where

$$
\Omega_{1}:=\operatorname{gph} G, \quad \Omega_{2}:=\operatorname{gph}(-Q), \quad \Omega_{3}:=\Omega \times Y .
$$


We intend to derive all the conditions of this theorem ensuring the existence of weak minimizers to the SOPEC problem (1.3) from the corresponding conditions of Theorem 3.2 applied to problem (3.16). It is clear from the latter conditions that the main task in this procedure is to express the basic normal cone to the intersection set $\Xi$ from (3.11) and the SNC property of this set via the normal cone to the sets $\Omega_{i}$ defined in (3.15) and the SNC property for these sets. In what follows we do it by applying appropriate results of the generalized differential and SNC calculi developed in [7].

Applying first the intersection rule for basic normals from [7, Corollary 3.37] to the intersection of $n=3$ sets in (3.11) at $v:=(x, y) \in \Xi$ and get the inclusion

$$
N(v ; \Xi) \subset N\left(v ; \Omega_{1}\right)+N\left(v ; \Omega_{2}\right)+N\left(v ; \Omega_{3}\right)
$$

provided that all but one of these sets are SNC at $v$ that the normal qualification condition

$$
\left[v_{i}^{*} \in N\left(v ; \Omega_{i}\right), \quad v_{1}^{*}+v_{2}^{*}+v_{3}^{*}\right] \Longrightarrow v_{i}^{*}=0 \text { for } i=1,2,3
$$

is satisfied. Furthermore, by [7, Corollary 3.81] the intersection set $\Xi$ is $S N C$ at $v$ if the qualification condition (3.18) holds and all the sets $\Omega_{1}, \Omega_{2}, \Omega_{3}$ are SNC at this point.

Taking into account the structures of the sets $\Omega_{i}$ in (3.16), definition (2.3) of the normal coderivative, and the relationship

$$
\left(x^{*}, y^{*}\right) \in N((x,-y) ; \operatorname{gph}(-Q)) \Longleftrightarrow\left(x^{*},-y^{*}\right) \in N((x, y) ; \operatorname{gph} Q)
$$

we conclude that the normal qualification condition (3.18) is equivalent to the qualification condition (3.13) of the theorem. Observe also that by (3.16) and (3.17) the inclusion $\left(x^{*}, 0\right) \in N((x, y) ; \Xi)$ implies the description

$$
x^{*} \in D_{N}^{*} G(x, y)\left(y^{*}\right)+D_{N}^{*} Q(x,-y)\left(y^{*}\right)+N(x ; \Omega)
$$

with some $y^{*} \in Y^{*}$. Substituting this into the qualification condition (3.7) of Theorem 3.2 with the cost mapping $\tilde{F}(x, y)=F(x)$, we get the qualification condition (3.12). Finally, substituting (3.19) into the Palais-Smale condition of Theorem 3.2, we arrive at the SOPEC Palais-Smale condition (3.14) and complete the proof of the theorem.

Remark 3.5 (existence of optimal solutions for specific classes of SOPECs). Similarly to Corollary 3.3 of Theorem 3.2, we conclude that the qualification condition (3.12) and the SNC requirement (c) of Theorem 3.4 hold automatically if the cost mapping $F$ in (1.3) is epi-Lipschitz-like around points $(x, z) \in \operatorname{gph} F$ satisfying the constraints in (1.3). Of course, the SNC assumptions are not needed at all in Theorem 3.4 if the spaces $X$ and $Y$ (while not $Z$ ) are finite-dimensional. Observe also that if $\Omega=X$ in (1.3), i.e., this problem contains only equilibrium but not geometric constraints, that the qualification condition (3.13) of Theorem 3.4 reduces to the Fredholm qualification condition

$$
\left[0 \in D_{N}^{*} G(x, y)\left(y^{*}\right)+D_{N}^{*} Q(x,-y)\left(y^{*}\right)\right] \Longrightarrow y^{*}=0
$$

which means that the adjoint generalized equation to (1.1) has only the trivial solution. 


\section{Necessary Optimality Condition for SOPECs}

In the last section of the paper we derive necessary optimality conditions for local optimal solutions to the SOPECs under consideration. We pay the main attention to the case of usual local minimizers to (1.3), which requires less restrictive assumptions. At the same time, our approach based on the extremal principle allows us to derive similar optimality conditions for weak local minimizers under the additional assumption on the nonempty interior of the ordering cone $\Theta$.

Proceeding similarly to Section 3 , we start with the set-valued optimization problem (3.1) involving only the geometric constraints. We say that $(\bar{x}, \bar{z})$ is a local minimizer to (3.1) if $\bar{x} \in \Xi, \bar{z} \in F(\bar{x})$, and there is a neighborhood $U$ of $\bar{x}$ such that (3.2) holds with the replacement of $F(\Xi)$ by $F(\Xi \cap U)$. The notion of weak local minimizers to (3.1) is defined in the same way by the substitution of $F(\Xi \cap U)$ instead of $F(\Xi)$ in (3.3) provided that $\operatorname{int} \Theta \neq \emptyset$. The next theorem gives necessary conditions for local minimizers to (3.1).

Theorem 4.1 (necessary conditions in set-valued optimization with geometric constraints). Let $(\bar{x}, \bar{z})$ be a local minimizer to the constrained set-valued optimization problem (3.1), where the convex cone $\Theta$ is SNC at the origin and where the sets epi $F$ and $\Xi$ are locally closed around $(\bar{x}, \bar{z})$ and $\bar{x}$, respectively. Assume also that either $F$ is $S N E C$ at $(\bar{x}, \bar{z})$ or $\Xi$ is $S N C$ at $\bar{x}$ and that the qualification condition

$$
\partial_{M}^{\infty} F(\bar{x}, \bar{z}) \cap(-N(\bar{x} ; \Xi))=\{0\}
$$

is satisfied; the latter group of assumptions is automatic if $F$ is $E L L$ around $(\bar{x}, \bar{z})$. Then

$$
0 \in \partial_{N} F(\bar{x}, \bar{z})+N(\bar{x} ; \Xi)
$$

Proof. We proceed by creating the extremal system of sets generated by the local minimizer $(\bar{x}, \bar{z})$ to $(3.1)$ and then by using the extremal principle. Define the sets

$$
\Omega_{1}:=\operatorname{epi} F, \quad \Omega_{2}:=\Xi \times(\bar{z}-\Theta)
$$

in the (Asplund) product space $X \times Z$ endowed with the sum norm $\|(x, z)\|:=\|x\|+\|z\|$ and show that $(\bar{x}, \bar{z})$ is a local extremal point of the system $\left\{\Omega_{1}, \Omega_{2}\right\}$. We obviously have $(\bar{x}, \bar{z}) \in \Omega_{1} \cap \Omega_{2}$, where the sets $\Omega_{1}$ and $\Omega_{2}$ are locally closed around this point. To justify condition (2.10) for the set system (4.3), we find a neighborhood $U$ of $\bar{x}$ by the local minimality of $(\bar{x}, \bar{z})$ to $(3.1)$ such that

$$
F(\Xi \cap U) \cap(\bar{z}-\Theta)=\{\bar{z}\}
$$

Pick any $c \in \Theta \backslash\{0\}$ and define a sequence $\left\{c_{k}\right\} \subset Z$ by $c_{k}:=k^{-1} c$. Let us show that

$$
\Omega_{1} \cap\left(\Omega_{2}-\left(0, c_{k}\right)\right) \cap(U \times Z)=\emptyset, \quad k \in \mathbb{N},
$$

which means the fulfillment of (2.10) along the sequence of $a_{k}:=-\left(0, c_{k}\right) \downarrow 0$ as $k \rightarrow \infty$ with $V:=U \times Z$. Arguing by contradiction, suppose that (4.5) does not hold, i.e.,

$$
\text { there is }(x, z) \in U \times Z \text { with }(x, z) \in \Omega_{1} \cap\left(\Omega_{2}-\left(0, c_{k}\right)\right)
$$


By the construction of sets (4.3), we have

$x \in \Xi$ and $z \in F(x)+\xi$ with $\xi \in \Theta$ and $z+c_{k} \in \bar{z}-\Theta, \quad k \in \mathbb{N}$

Thus $z-\xi \in F(\Xi \cap U)$ and $z-\xi \in \bar{z}-c_{k}-\Theta \subset(\bar{z}-\Theta) \backslash\{\bar{z}\}$, where the latter inclusion holds due to the pointedness of $\Theta$. This implies $\bar{z} \neq z-\xi \in F(\Xi \cap U) \cap(\bar{z}-\Theta)$, which contradicts (4.4) and justifies the extremality property (4.5) of sets (4.3) at the point $(\bar{x}, \bar{z})$.

Employing now the extremal principle to this set system, we find sequences $\varepsilon_{k} \downarrow 0$, $\left(x_{1 k}, z_{1 k}\right) \in$ epi $F,\left(x_{2 k}, z_{2 k}\right) \in \Xi \times(\bar{z}-\Theta)$ with $\left\|\left(x_{i k}, z_{i k}\right)-(\bar{x}, \bar{z})\right\| \leq \varepsilon_{k}$ for $i=1,2$ satisfying the relationships

$$
\begin{aligned}
& \left(x_{1 k}^{*},-z_{1 k}^{*}\right) \in \widehat{N}\left(\left(x_{1 k}, z_{1 k}\right) ; \text { epi } F\right), \quad x_{2 k}^{*} \in \widehat{N}\left(x_{2 k} ; \Xi\right), \quad-z_{2 k}^{*} \in \widehat{N}\left(\bar{z}-z_{2 k} ; \Theta\right), \\
& \left\|\left(x_{1 k}^{*}, z_{1 k}^{*}\right)+\left(x_{2 k}^{*}, z_{2 k}^{*}\right)\right\| \leq \varepsilon_{k}, \quad 1-\varepsilon_{k} \leq\left\|\left(x_{1 k}^{*}, z_{1 k}^{*}\right)\right\|+\left\|\left(x_{2 k}^{*}, z_{2 k}^{*}\right)\right\| \leq 1+\varepsilon_{k} .
\end{aligned}
$$

By (4.7) and the Asplund property of $X \times Z$, we assume without loss of generality that the bounded sequences $\left\{\left(x_{1 k}^{*}, z_{1 k}^{*}\right)\right\}$ and $\left\{\left(x_{2 k}^{*}, z_{2 k}^{*}\right)\right\}$ weak $k^{*}$ converge in $X^{*} \times Z^{*}$ to $\left(x^{*},-z^{*}\right)$ and $\left(-x^{*}, z^{*}\right)$, respectively. Passing to the limit in (4.6) as $k \rightarrow \infty$ and the basic definitions of Section 2 justify the inclusions

$$
x^{*} \in D_{N}^{*} \mathcal{E}_{F}(\bar{x}, \bar{z})\left(z^{*}\right), \quad-x^{*} \in N(\bar{x} ; \Xi), \quad-z^{*} \in N(0 ; \Theta)
$$

Let us finally show that, due to the SNC and qualification assumptions of the theorem, $z^{*} \neq 0$, i.e., we get $\left\|z^{*}\right\|=1$ by rescaling. Assuming the contrary, we have from above that

$$
z_{2 k}^{*} \stackrel{w^{*}}{\rightarrow} 0 \text { as } k \rightarrow \infty \text { with. }-z_{2 k}^{*} \in \widehat{N}\left(\bar{z}-z_{2 k} ; \Theta\right), \quad k \in \mathbb{N},
$$

which yields $\left\|z_{2 k}^{*}\right\| \rightarrow 0$ by the SNC property of $\Theta$ at the origin. This implies by (4.7) that $\left\|z_{1 k}^{*}\right\| \rightarrow 0$ as $k \rightarrow \infty$. Furthermore, from (4.6) and (2.7) we conclude that $x^{*} \in \partial_{M}^{\infty} F(\bar{x}, \bar{z})$; and thus $x^{*}=0$ due to (4.8) and the qualification condition (4.1). Therefore

$$
x_{1 k}^{*} \stackrel{w^{*}}{\rightarrow} 0 \text { and } x_{2 k}^{*} \stackrel{w^{*}}{\rightarrow} 0 \text { as } k \rightarrow \infty
$$

The latter relationships imply that either $\left\|x_{1 k}^{*}\right\| \rightarrow 0$ or $\left\|x_{2 k}^{*}\right\| \rightarrow 0$ as $k \rightarrow \infty$ depending on the alternative SNEC/SNC assumption on $F$ and $\Theta$ imposed in the theorem. Involving now both relationships in (4.7), we arrive at a contradiction with the nontriviality of $\left(x_{i k}^{*}, z_{i k}^{*}\right)$ for $i=1,2$ and all $k \in \mathbb{N}$ sufficiently large. Thus $z^{*} \neq 0$ and $x^{*} \in \partial_{N} F(\bar{x}, \bar{z})$ by $(2.5)$ and (4.8). Recalling that $-x^{*} \in N(\bar{x} ; \Xi)$ in (4.8), we complete the proof of the theorem.

If the cost mapping $F$ in (3.1) is single-valued, the necessary optimality conditions of Theorem 4.1 reduce to those in [2, Theorem 5.1] derived by another approach based on the subdifferential variational principle from [7, Theorem 2.28]. However, the realization of the latter approach requires additional assumptions of the type imposed in Theorem 3.2, which are not needed in Theorem 4.1. 
Remark 4.2 (necessary conditions for weak local minimizers). All the conditions of Theorem 4.1 hold with no change for weak local minimizers $(\bar{x}, \bar{z})$ to problem (3.1) provided that int $\Theta \neq \emptyset$. The only change needed in the proof given above is to show that $(\bar{x}, \bar{z})$ is a local extremal point of the set system (4.3) when the local minimum relation (4.4) is replaced by the weak local minimum property

$$
F(\Xi \cap U) \cap(\bar{z}-\operatorname{int} \Theta)=\emptyset
$$

To justify this, we pick any $c \in \operatorname{int} \Theta$ and proceed exactly as in the proof of Theorem 4.1 showing that (4.9) implies the extremality relation (4.5) arguing by contradiction.

Finally in this section, we derive necessary optimality conditions for local minimizers to the SOPEC problem (1.3) involving geometric and equilibrium constraints by reducing them to just the geometric ones as in the proof of Theorem 3.2. The case of weak local minimizers to SOPECs can be treated similarly based on Remark 4.2.

Theorem 4.3 (necessary conditions for local minimizers to SOPECs). Let $(\bar{x}, \bar{z})$ be a local minimizer to the SOPEC (1.3), and let $\bar{y} \in G(\bar{x}) \cap(-Q(\bar{x}))$. Assume that the ordering cone $\Theta$ is $S N C$ at the origin and that all the sets epi $F, \operatorname{gph} G, \operatorname{gph} Q$ and $\Omega$ are locally closed at $(\bar{x}, \bar{z}) ;(\tilde{x}, \bar{y}),(\tilde{x},-\bar{y})$ and $\bar{x}$, respectively. Suppose also the following two qualification conditions

$$
\begin{aligned}
& \left(-\partial_{M}^{\infty} F(\bar{x}, \bar{z})\right) \cap\left(D_{N}^{*} G(\bar{x}, \bar{y})\left(y^{*}\right)+D_{N}^{*} Q(\bar{x},-\bar{y})\left(y^{*}\right)+N(\bar{x} ; \Omega)\right)=\{0\} \\
& {\left[\begin{array}{l}
x_{1}^{*} \in D_{N}^{*} G(\bar{x}, \bar{y})\left(y^{*}\right), x_{2}^{*} \in D_{N}^{*} Q(\bar{x},-\bar{y})\left(y^{*}\right) \\
x_{3}^{*} \in N(\bar{x} ; \Omega), \quad x_{1}^{*}+x_{2}^{*}+x_{3}^{*}=0
\end{array}\right] \Longrightarrow\left[\begin{array}{l}
y^{*}=0 \\
x_{1}^{*}=x_{2}^{*}=x_{3}^{*}=0
\end{array}\right]}
\end{aligned}
$$

are satisfied whenever $y^{*} \in Y^{*}$ and that one of the following SNC conditions holds:

- either $F$ is partially SNEC at $(\bar{x}, \bar{z})$ and all but one of the sets $\operatorname{gph} G, \operatorname{gph} Q$, and $\Omega$ are $S N C$ at $(\bar{x}, \bar{z}),(\bar{x},-\bar{z})$, and $\bar{x}$, respectively;

-all the sets $\operatorname{gph} G, \operatorname{gph} Q$, and $\Omega$ are $S N C$ at the afore-mentioned reference points.

Then there is $y^{*} \in Y^{*}$ such that

$$
0 \in \partial_{N} F(\bar{x}, \bar{z})+D_{N}^{*} G(\bar{x}, \bar{y})\left(y^{*}\right)+D_{N}^{*} Q(\bar{x},-\bar{y})\left(y^{*}\right)+N(\bar{x} ; \Omega)
$$

Proof. Similarly to the proof of Theorem 3.4 we consider the set-valued optimization problem (3.15) with the cost mapping $\widetilde{F}(x, y):=F(x)$ and with only the geometric constraint $(x, y) \in \Xi$ defined by the set intersection

$$
\Xi:=\Omega_{1} \cap \Omega_{2} \cap \Omega_{3} \subset X \times Y
$$

where the sets $\Omega_{1}, \Omega_{2}$, and $\Omega_{3}$ are given in (3.16). Applying the results of Theorem 4.1 to the latter problem and then using the normal cone and SNC intersection rules from [7] for the above set $\Xi$ as in the proof of Theorem 3.4, we arrive at the necessary optimality condition (4.10) under the qualification and SNC assumptions of the theorem. 
Remark 4.4 (some particular cases). Equilibrium constraints of the type

$$
0 \in G(x)+Q(x)
$$

in (1.3) contain as particular cases virtually all the types of constraints considered in the literature. Thus the necessary optimality conditions of Theorem 4.3 improve and unify various results previously known in this direction. Let us mention some particular cases referring the reader to $[1,2,8]$ for more examples and discussions.

- Inequality constraints: $\varphi_{i}(x) \leq 0, i=1, \ldots, m$, corresponding to (4.11) with

$$
G(x)=\prod_{i=1}^{m}\left[\varphi_{i}(x), \infty\right), \quad Q(x)=\mathbb{R}_{\geq}^{m}
$$

- Equality constraints: $\varphi_{i}(x)=0, i=m+1, \ldots, m+r$, corresponding to (4.11) with

$$
G(x)=\left(\varphi_{m+1}(x), \ldots, \varphi_{m+r}(x)\right), \quad Q(x)=\{0\} \in \mathbb{R}^{r}
$$

- Operator, constraints: $x \in G^{-1}(\Lambda)$ defined by a set-valued mapping $G: X \Rightarrow Y$ and a set $\Lambda \subset Y$. These constraints are equivalently written as $G(x) \cap \Lambda \neq \emptyset$ and correspond to the equilibrium constraints (4.11) given by the same mapping $G$ and $Q(x)=-\Lambda$. Necessary optimality conditions for set-valued optimization problems with constraints of the latter type have been recently derived in [18] under certain normal compactness assumptions on the set $\Lambda$, which exclude the case when $\Lambda$ is a singleton; in particular, they do not cover the case of the inclusion constraints $0 \in G(x)$, which is well handled by Theorem 4.3.

- Fixed-point constraints: $x \in Q(x)$ with $Q: X \rightrightarrows Y$. They correspond to the equilibrium constraints (4.11) defined by the same mapping $Q$ and $G(x)=-x$.

Remark 4.5 (equilibrium constraints in composite subdifferential forms). Many classes of equilibrium constraints important for both optimization/equilibrium theory and applications can be described in one of the following composite subdifferential forms:

$$
\begin{aligned}
& 0 \in G(x)+\partial(\varphi \circ q)(x) \\
& 0 \in G(x)+(\partial \varphi \circ q)(x)
\end{aligned}
$$

with $q: X \rightarrow Y, \varphi: Y \rightarrow \mathbb{R}$, and $G: X \rightrightarrows Y^{*}$. Such systems include nonlinear and implicit complementarity problems, variational and hemivariational inequalities of different kinds and their generalizations, KKT systems, mechanical and economic equilibria, etc.; see the books $[4,7,8,15]$ for more details and examples. The existence theorems and necessary optimality conditions obtained in this paper can be specified for the equilibrium systems of types (4.12) and (4.13) by using the corresponding second-order subdifferentials for extended-real-valued functions $\varphi$ in (4.12) and (4.13) and appropriate subdifferential and coderivative calculus rules; $c f .[7,8,9]$. 


\section{References}

[1] T. Q. Bao, P. Gupta, B. S. Mordukhovich: Necessary conditions in multiobjective optimization with equilibrium constraints. J. Optim. Theory Appl. 134 (2007), No. 3.

[2] T. Q. Bao, B.S. Mordukhovich: Variational principles for set-valued mappings with applications to multiobjective optimization. Control and Cybernetics, to appear.

[3] J. M. Borwein, Q. J. Zhu: Techniques of Variational Analysis. CMS Books in Math., Vol. 20, Springer, New York, 2005.

[4] F. Facchinei, J.-S. Pang: Finite-Dimensional Variational Inequalities and Complementary Problems Springer, New York, 2003.

[5] J. Jahn: Vector Optimization: Theory, Applications and Extensions. Series Oper. Res., Springer, Berlin, 2004.

[6] Z.-Q. Luo, J.-S. Pang, D. Ralph: Mathematical Programs with Equilibrium Constraints. Cambridge University Press, Cambridge, UK, 1996.

[7] B. S. Mordukhovich: Variational Analysis and Generalized Differentiation, I: Basic Theory. Grundlehren Series (Fundamental Principles of Mathematical Sciences), Vol. 330 Springer, Berlin, 2006.

[8] B. S. Mordukhovich: Variational Analysis and Generalized Differentiation, II: Applications. Grundlehren Series (Fundamental Principles of Mathematical Sciences), Vol. 331, Springer, Berlin, 2006.

[9] B. S. Mordukhovich, J. V. Outrata: Second-order subdifferentials and their applications. SIAM J. Optim. 12 (2001), 139-169.

[10] B. S. Mordukhovich, J. V. Outrata: Coderivative analysis of quasivariational inequalities with applications to stability and optimization. SIAM J. Optim., to appear.

[11] B. S. Mordukhovich, J. V. Outrata, M. Červinka: Equilibrium problems with complementarity constraints: case study with applications to oligopolistic markets. Optimization, to appear.

[12] J. V. Outrata: Optimality conditions for a class of mathematical programs with equilibrium constraints. Math. Oper. Res. 24 (1999), 627-644.

[13] J. V. Outrata: A generalized mathematical program with equilibrium constraints. SIAM J. Control Optim. 38 (2000), 1623-1638.

[14] J. V. Outrata: A note on a class of equilibrium problems with equilibrium constraints. Kybernetika 40 (2004), 585-594.

[15] J. V. Outrata, M. Kočvara, J. Zowe: Nonsmooth Approach to Optimization Problems with Equilibrium Constraints. Kluwer Academic Publishers, Dordrecht, The Netherlands, 1998.

[16] S. M. Robinson: Generalized equations and their solutions, I: basic theory. Math. Prog. Study 10 (1979), 128-141.

[17] R. T. Rockafellar, R. J.-B. Wets: Variational Analysis. Grundlehren Series (Fundamental Principles of Mathematical Sciences), Vol. 317, Springer, Berlin, 1998.

[18] X. Y. Zheng, K. F. Ng: The Lagrange multiplier rule for multifunctions in Banach spaces. SIAM J. Optim. 17 (2006), 1154-1175. 